\title{
Wilms tumor 1 gene, CD97, and the emerging biogenetic profile of glioblastoma
}

\author{
Aravind Somasundaram, B.S., ${ }^{1}$ Nathan Ardanowski, B.S., ${ }^{1}$ Charles F. Opalak, M.P.H., ${ }^{1}$ \\ Helen L. Fillmore, Ph.D., ${ }^{2}$ Archana Chidambaram, M.D., Ph.D., 3 \\ and William C. Broaddus, M.D., Ph.D. ${ }^{1}$ \\ ${ }^{1}$ Department of Neurosurgery, Virginia Commonwealth University, Richmond, Virginia; ${ }^{3}$ Department of \\ Hematology-Oncology, Roswell Park Cancer Institute, Buffalo, New York; and ${ }^{2}$ School of Pharmacy and \\ Biomedical Sciences, University of Portsmouth, Portsmouth, United Kingdom
}

\begin{abstract}
Glioblastoma multiforme (GBM) is the most common type of primary brain tumor, and current treatment regimens are only marginally effective. One of the most vexing and malignant aspects of GBM is its pervasive infiltration into surrounding brain tissue. This review describes the role of the Wilms tumor 1 gene (WT1) and its relationship to GBM. WT1 has several alternative splicing products, one of which, the KTS ${ }^{+}$variant, has been demonstrated to be involved in the transcriptional activation of a variety of oncogenes as well as the inhibition of tumor suppressor genes. Further, this paper will examine the relationship of WT1 with CD97, a gene that codes for an epidermal growth factor receptor family member, an adhesion G-protein-coupled receptor, thought to promote tumor invasiveness and migration. The authors suggest that further research into $W T 1$ and $C D 97$ will allow clinicians to begin to deal more effectively with the infiltrative behavior displayed by GBM and design new therapies that target this deadly disease. (http://thejns.org/doi/abs/10.3171/2014.9.FOCUS14506)
\end{abstract}

KeY Words • glioblastoma • Wilms tumor 1 gene • CD97

$\mathrm{G}$ LIOBLASTOMA multiforme (GBM), a WHO Grade IV glioma, is the most common primary malignancy in the central nervous system. GBM is characterized by high degrees of parenchymal invasion, vascularization secondary to angiogenesis, necrosis, and de-differentiation. Due to its aggressive nature, GBM carries a poor prognosis, with $35.7 \%$ survival at 1 year and $4.7 \%$ survival at 5 years. ${ }^{26}$ Currently, the optimal treatment paradigm is aggressive resection followed by radiotherapy and concomitant chemotherapy. ${ }^{33}$ Despite intensive laboratory and clinical research, only moderate advances have been made in improving the quality of life in GBM patients. This suggests that there is a need to build a complete biological profile to uncover novel molecular targets that can be employed in future therapies. Recently, our laboratory and others have shifted their fo-

Abbreviations used in this paper: DAF = decay accelerating factor; EGF-TM7 = epidermal growth factor-7 transmembrane; GBM = glioblastoma multiforme; shRNA = short hairpin RNA; WTAP = Wilms tumor 1-associated protein (pre-mRNA-splicing regulator WTAP); WT1 = Wilms tumor 1 . cus to the Wilms tumor 1 gene $(W T 1) .^{5-8}$ WT1 was first isolated in 1990 by Haber et al. with their discovery that an internal deletion within an 11 p13 zinc finger gene contributes to the development of Wilms tumor, a pediatric kidney malignancy. ${ }^{10}$ WT1 was initially thought to be a tumor suppressor gene, but subsequent research uncovered its oncogenic role when it was demonstrated that WT1 can suppress hTERT gene expression and telomerase activity in clear cell renal cell carcinoma. ${ }^{31}$

Menssen et al. were the first group to show the expression of WT1 in human GBM. They reported a high WT1 expression in $63 \%$ of GBM cell lines. ${ }^{21}$ In the same year, we also reported the expression of WT1 in a variety of ovarian and GBM cultured cell lines (Quezado MM, Dechsukhum C, Garrett CT, et al., presented at the United States and Canadian Academy of Pathology Annual Meeting, 2000). Since these initial descriptions, there has been an expanding interest in connecting WT1 and its oncological effect with GBM. This paper will review the studies to date that have analyzed the relationship of WTI to GBM and describe a novel G-protein coupled receptor, CD97, which may play a role in GBM invasion. 


\section{A. Somasundaram et al.}

\section{Wilms Tumor 1 Gene}

Though WT1 is absent in normal neural tissue, it has been described in cultured neoplastic glial cell lines, and further work has indicated high WTI expression in acute myeloid leukemia and acute lymphoid leukemia., ${ }^{715}$ WTI encodes for a zinc finger family transcription factor located at $11 \mathrm{p} 13$ whose physiological expression is associated with normal genitourinary embryologic development. ${ }^{1}$ It has been hypothesized that WTI may exert its oncological effects in a multifaceted modality via an alternative mRNA splicing variant that involves the insertion of the 3-amino acid sequence of lysine, threonine, serine (KTS) into the active zinc finger region. ${ }^{28}$ This is in contrast to the $\mathrm{KTS}^{-}$variant, which transcriptionally regulates suppression of at least 7 putative tumor suppressor genes and increases expression of 11 reputed oncogenes. ${ }^{5} \mathrm{The} \mathrm{KTS}^{+}$ product, on the other hand, has only a limited role in direct transcriptional regulation; instead it participates in putative splicing factor interactions and has an association with olfactory neuron development. ${ }^{14}$

\section{GBM and Wilms Tumor}

When WTl expression was first reported in brain tumors, it was hypothesized that testing for $W T 1$ expression might not have clinical significance..$^{21}$ The first laboratory investigation that focused on WTI expression in central nervous system malignancies, including GBM, found WTI expression in most of these tumors; yet there were no mutations affecting the zinc fingers of the gene product in tumors expressing WT1. ${ }^{9}$ Though this appeared to indicate that $W T 1$ expression might not have clinical relevance to the molecular etiology of brain tumors, these results nonetheless ignited a novel interest in the relationship of WTI to central nervous system tumors. A few years later, Oji et al. were able to show that the WT1 protein expression was significantly greater in high-grade tumors than in low-grade tumors and that treatment with WT1 antisense oligomers specifically inhibited GBM cell lines. ${ }^{24}$ This study, coupled with later confirmation from immunohistochemical tests that demonstrated WT1 expression in GBM cells, highlighted its potential as a target for immunotherapy. ${ }^{12,23}$ This differential expression of WTl in high-grade brain tumors has recently been validated in an in vivo study analyzing human gliomas, which included 442 glioblastomas, 303 astrocytomas, 41 oligodendrogliomas, and 43 oligoastrocytomas. ${ }^{27}$ The results showed that $W T 1$ expression in brain tumors increased with WHO grade, older age, and absence of $\mathrm{IDH} 1$ mutation. $^{27}$

The introduction of a WT1-based vaccine in clinical trials was first described by Oka et al. in 2004..$^{13,25}$ The authors reported the effects of the vaccine on breast cancer, lung cancer, and leukemia in 26 patients, reporting tumor regression and no toxicity as a result of the drug. The same group recently published data on a Phase II clinical trial that included 21 patients who received WT1 peptide vaccination for recurrent GBM. ${ }^{15}$ Preliminary data showed that patients with WT1/HLA-A*2402 positivity who received WT1 immunotherapy showed a favorable outcome when compared with patients treated with a standard chemotherapy protocol..$^{15}$ In a follow-up study analyzing the same GBM patient population, the authors reported that $W T 1$ expression can be used as a prognostic marker in determining progression-free survival after immunotherapy and that patients with intermediate WTI expression levels may have the best outcomes. ${ }^{4}$

One possible mechanism of action of a WT1-based vaccination could lie in its suppression of WT1's inactivation of tumor suppressor gene p53 (TP53). This hypothesis was initially tested in p53-null Saos-2 osteosarcoma cells, and it was revealed that WT1 inhibits p53-mediated apoptosis, which is normally induced by chemotherapy, radiation, and overexpression of wild-type $\mathrm{p} 53 .{ }^{20}$ Clark et al. continued to investigate this relationship between WT1 and p53 in GBM by examining the effect of WT1 expression and WT1 silencing on p53-mediated cell death and response to radiotherapy. ${ }^{6}$ Results showed that $W T 1$ silencing led to GBM cells becoming susceptible to radiation-induced death, suggesting a potential target to improve responses to radiotherapy. The relationship of WT1 silencing and chemotherapy was tested in a separate study by Chen et al. that revealed similar results; WT1 silencing resulted in increased chemotherapeutic response. ${ }^{3}$ It was reported in follow-up work that there was an interaction between the $\mathrm{KTS}^{+}$isoform of WT1 and wild-type p53, revealing that the potential oncogenic function of WTI in GBM may depend on this splicing variant.?

Additional avenues whereby $W T 1$ promotes tumorigenesis was found in WTI's relation with Wilms tumor 1-associated protein (WTAP, also known as pre-mRNAsplicing regulator WTAP). WTAP is a nuclear protein that was isolated through a yeast 2-hybrid screen and has been reported to be overexpressed in GBM cell lines. ${ }^{16,19} \mathrm{Jin}$ et al. suggested that WTAP may regulate migration and invasion of GBM cells by controlling epidermal growth factor signaling. ${ }^{16}$ The link between $W T l$ and cancer cell invasion was initially reported by Jomgeow et al. in TYK ovarian cancer cell lines, where in vitro experiments demonstrated that $W T 1$ overexpression led to an increase in cell invasion. ${ }^{17}$ Kijima et al. demonstrated that GBM cell lines with downregulated WTI expression had decreased tumorigenicity in an intracranial in vivo assay. ${ }^{18}$ Further, they suggested that WTI may play a role in apoptosis as cell lines transfected with anti-WTI shRNA showed upregulation of apoptosis-related genes when compared with control cell lines-supporting a previous report that WT1 may be involved in apoptosis in GBM. ${ }^{34}$ These preliminary studies suggest that the oncogenic role WTI may play in GBM cell proliferation, invasiveness, and survival involves suppressed apoptosis.

\section{CD97}

Recent research at our institution has focused on another molecule that appears to be involved in promoting the invasiveness of GBM through a relationship with $W T 1$, as an apparent target of WT1 transcriptional activation. ${ }^{5}$ The CD97 molecule, also known as TM7LN1, is a member of the epidermal growth factor-7 transmembrane (EGF-TM7) cell receptor subfamily that mediates cell-cell interactions. CD97 is a part of a 6-mem- 
ber family of adhesion G-protein coupled receptors that have been primarily reported as expressed on the surface of leukocytes. ${ }^{29}$ Structurally, CD97 resembles all other EGF-TM7 receptors, consisting of an extracellular alpha unit, a transmembrane beta unit composed of 7 domains, and an intracellular C-terminus. The alpha unit is composed of 5 EGF-like domains and a single Arg-Gly-Asp motif, which acts as a binding site for several classes of integrins. Alternative splicing of the mRNA transcript of the alpha subunit allows 3 isoforms to be generatedEGF (1-5), EGF $(1,2,5)$, and EGF $(1,2,3,4,5)$ - which allows binding heterogeneity. ${ }^{30} \mathrm{CD} 97$ has 3 known ligands, which include CD55/decay accelerating factor (DAF), chondroitin sulfate, and alpha-beta integrin. CD55/DAF serves as an inhibitor of the complement system and binds to the EGF $(1,2,5)$ domain. ${ }^{11,22,30}$ Chondroitin sulfate proteoglycans are components of the extracellular matrix that have a role in cell adhesion, growth, receptor binding, and the migration of cells and binding to EGF $(1,2,3,4,5)$ domains. ${ }^{29}$ Integrins, which are transmembrane receptors involved in cell-to-cell adhesion, migration, and signaling, bind to $\operatorname{EGF}(1,2,5)$ and $\operatorname{EGF}(1,2,3,4,5) .{ }^{29}$ The capability of these various isoforms to bind to ligands with complex functions makes CD97 an intriguing therapeutic target for cancer treatment, because it raises the possibility that an exogenous small molecule might be developed that could inhibit its function.

CD97 was first described in thyroid carcinomas, showing a high expression in undifferentiated anaplastic carcinomas. ${ }^{2}$ Since this initial description in thyroid cancer, further work has found CD97 to be highly expressed in pancreatic cancers, colon cancers, and oral squamous cell carcinoma. ${ }^{32}$ Chidambaram et al. were the first to describe CD97 in GBM. ${ }^{5}$ They showed that CD97 expression was significantly downregulated in all 3 GBM cell lines they analyzed after suppression of WT1 expression. They observed that decreasing the endogenous expression of CD97 decreased the ability of cells to invade through Matrigel, suggesting that CD97 might promote cellular invasiveness. Safaee et al., using human GBM cell lines prepared at their institution, revealed through siRNA knockdown that CD97 may play a role in invasiveness and migration but not proliferation..$^{30}$ They reported that patients whose tumors overexpressed CD97 had a significantly shorter survival time when compared with those who had tumors that showed a downregulation of $C D 97$ using data from the Cancer Genome Atlas database. ${ }^{30}$ The analysis included 212 patients and revealed that patients with upregulation of $C D 97$ had a median survival of 250 days compared with a median of 500 days for patients with downregulated expression. This preliminary study suggests that upregulation of $C D 97$ in GBM patients may confer a poorer prognosis, and future studies will help further elucidate this relationship.

\section{Conclusions}

When WT1 was first reported in brain tumors it was hypothesized that it was unlikely to be of clinical significance, but as research has evolved WT1 has been shown to play a significant role in GBM tumor biology. WT1 pro- motion of tumor invasiveness, proliferation, and survival through its interaction with p53, WTAP, and CD97 all contribute to its multifaceted oncogenic role. Molecular genetics is opening exciting new avenues in the understanding of difficult-to-treat cancers such as GBM. In an effort to capitalize on this ongoing revolution, this paper has summarized the current knowledge concerning WT1 and GBM and this gene's potential role in tumor biology. Moving forward, the authors call upon researchers to continue to elucidate the role of the $\mathrm{KTS}^{+} \mathrm{WT} 1$ variant in tumorigenesis, further define the interplay between WT1 and $C D 97$, and continue to develop novel therapies using these new targets. Future studies that will help further develop this emerging field of glioblastoma biology include: larger experiments that use human tissue to clinically correlate WT1 and CD97 expression and patient outcomes, identification of molecules that can inhibit CD97 to see if there is a survival advantage that could lead to therapeutic options for GBM patients, investigate WT1 and CD97 expression in recurrent GBM, and a study focusing on the microenvironment changes during GBM progression with and without treatment interventions to further investigate the multiple effects of CD97 in invasion and growth.

\section{Disclosure}

The authors report no conflict of interest concerning the materials or methods used in this study or the findings specified in this paper.

Author contributions to the study and manuscript preparation include the following. Conception and design: Broaddus, Somasundaram. Acquisition of data: Somasundaram, Opalak. Analysis and interpretation of data: Somasundaram, Ardanowski, Opalak. Drafting the article: all authors. Critically revising the article: all authors. Reviewed submitted version of manuscript: all authors. Approved the final version of the manuscript on behalf of all authors: Broaddus. Study supervision: Broaddus, Fillmore.

\section{References}

1. Armstrong JF, Pritchard-Jones K, Bickmore WA, Hastie ND, Bard JB: The expression of the Wilms' tumour gene, WT1, in the developing mammalian embryo. Mech Dev 40:85-97, 1993

2. Aust G, Eichler W, Laue S, Lehmann I, Heldin NE, Lotz O, et al: CD97: a dedifferentiation marker in human thyroid carcinomas. Cancer Res 57:1798-1806, 1997

3. Chen MY, Clark AJ, Chan DC, Ware JL, Holt SE, Chidambaram A, et al: Wilms' tumor 1 silencing decreases the viability and chemoresistance of glioblastoma cells in vitro: a potential role for IGF-1R de-repression. J Neurooncol 103:87-102, 2011

4. Chiba Y, Hashimoto N, Tsuboi A, Rabo C, Oka Y, Kinoshita $\mathrm{M}$, et al: Prognostic value of WT1 protein expression level and MIB-1 staining index as predictor of response to WT1 immunotherapy in glioblastoma patients. Brain Tumor Pathol 27:29-34, 2010

5. Chidambaram A, Fillmore HL, Van Meter TE, Dumur CI, Broaddus WC: Novel report of expression and function of CD97 in malignant gliomas: correlation with Wilms tumor 1 expression and glioma cell invasiveness. Laboratory investigation. J Neurosurg 116:843-853, 2012

6. Clark AJ, Chan DC, Chen MY, Fillmore H, Dos Santos WG, Van Meter TE, et al: Down-regulation of Wilms' tumor 1 expression in glioblastoma cells increases radiosensitivity independently of p53. J Neurooncol 83:163-172, 2007 


\section{A. Somasundaram et al.}

7. Clark AJ, Dos Santos WG, McCready J, Chen MY, Van Meter TE, Ware JL, et al: Wilms tumor 1 expression in malignant gliomas and correlation of +KTS isoforms with p53 status. J Neurosurg 107:586-592, 2007

8. Clark AJ, Ware JL, Chen MY, Graf MR, Van Meter TE, Dos Santos WG, et al: Effect of WT1 gene silencing on the tumorigenicity of human glioblastoma multiforme cells. Laboratory investigation. J Neurosurg 112:18-25, 2010

9. Dennis SL, Manji SS, Carrington DP, Scarcella DL, Ashley DM, Smith PJ, et al: Expression and mutation analysis of the Wilms' tumor 1 gene in human neural tumors. Int J Cancer 97:713-715, 2002

10. Haber DA, Buckler AJ, Glaser T, Call KM, Pelletier J, Sohn RL, et al: An internal deletion within an 11p13 zinc finger gene contributes to the development of Wilms' tumor. Cell 61:12571269,1990

11. Hamann J, Stortelers C, Kiss-Toth E, Vogel B, Eichler W, van Lier RA: Characterization of the CD55 (DAF)-binding site on the seven-span transmembrane receptor CD97. Eur J Immunol 28:1701-1707, 1998

12. Hashiba T, Izumoto S, Kagawa N, Suzuki T, Hashimoto N, Maruno M, et al: Expression of WT1 protein and correlation with cellular proliferation in glial tumors. Neurol Med Chir (Tokyo) 47:165-170, 2007

13. Hashimoto N, Tsuboi A, Chiba Y, Izumoto S, Oka Y, Yoshimine T, et al: [Immunotherapy targeting the Wilms' tumor 1 gene product for patients with malignant brain tumors.] Brain Nerve 61:805-814, 2009 (Jpn)

14. Hohenstein P, Hastie ND: The many facets of the Wilms' tumour gene, WT1. Hum Mol Genet 15:R196-R201, 2006

15. Izumoto S, Tsuboi A, Oka Y, Suzuki T, Hashiba T, Kagawa N, et al: Phase II clinical trial of Wilms tumor 1 peptide vaccination for patients with recurrent glioblastoma multiforme. J Neurosurg 108:963-971, 2008

16. Jin DI, Lee SW, Han ME, Kim HJ, Seo SA, Hur GY, et al: Expression and roles of Wilms' tumor 1-associating protein in glioblastoma. Cancer Sci 103:2102-2109, 2012

17. Jomgeow T, Oji Y, Tsuji N, Ikeda Y, Ito K, Tsuda A, et al: Wilms' tumor gene WT1 17AA(-)/KTS(-) isoform induces morphological changes and promotes cell migration and invasion in vitro. Cancer Sci 97:259-270, 2006

18. Kijima N, Hosen N, Kagawa N, Hashimoto N, Kinoshita M, Oji Y, et al: Wilms' tumor 1 is involved in tumorigenicity of glioblastoma by regulating cell proliferation and apoptosis. Anticancer Res 34:61-67, 2014

19. Little NA, Hastie ND, Davies RC: Identification of WTAP, a novel Wilms' tumour 1-associating protein. Hum Mol Genet 9:2231-2239, 2000

20. Maheswaran S, Englert C, Bennett P, Heinrich G, Haber DA: The WT1 gene product stabilizes p53 and inhibits p53-mediated apoptosis. Genes Dev 9:2143-2156, 1995

21. Menssen HD, Bertelmann E, Bartelt S, Schmidt RA, Pecher G, Schramm K, et al: Wilms' tumor gene (WT1) expression in lung cancer, colon cancer and glioblastoma cell lines compared to freshly isolated tumor specimens. J Cancer Res Clin Oncol 126:226-232, 2000

22. Mikesch JH, Schier K, Roetger A, Simon R, Buerger H,
Brandt B: The expression and action of decay-accelerating factor (CD55) in human malignancies and cancer therapy. Cell Oncol 28:223-232, 2006

23. Nakahara Y, Okamoto H, Mineta T, Tabuchi K: Expression of the Wilms' tumor gene product WT1 in glioblastomas and medulloblastomas. Brain Tumor Pathol 21:113-116, 2004

24. Oji Y, Suzuki T, Nakano Y, Maruno M, Nakatsuka S, Jomgeow T, et al: Overexpression of the Wilms' tumor gene W T1 in primary astrocytic tumors. Cancer Sci 95:822-827, 2004

25. Oka Y, Tsuboi A, Taguchi T, Osaki T, Kyo T, Nakajima H, et al: Induction of WT1 (Wilms' tumor gene)-specific cytotoxic T lymphocytes by WT1 peptide vaccine and the resultant cancer regression. Proc Natl Acad Sci U S A 101:13885-13890, 2004

26. Omuro A, DeAngelis LM: Glioblastoma and other malignant gliomas: a clinical review. JAMA 310:1842-1850, 2013

27. Rauscher J, Beschorner R, Gierke M, Bisdas S, Braun C, Ebner $\mathrm{FH}$, et al: WT1 expression increases with malignancy and indicates unfavourable outcome in astrocytoma. J Clin Pathol 67:556-561, 2014

28. Roberts SG: Transcriptional regulation by WT1 in development. Curr Opin Genet Dev 15:542-547, 2005

29. Safaee M, Clark AJ, Ivan ME, Oh MC, Bloch O, Sun MZ, et al: CD97 is a multifunctional leukocyte receptor with distinct roles in human cancers (review). Int J Oncol 43:1343-1350, 2013

30. Safaee M, Clark AJ, Oh MC, Ivan ME, Bloch O, Kaur G, et al: Overexpression of CD97 confers an invasive phenotype in glioblastoma cells and is associated with decreased survival of glioblastoma patients. PLoS ONE 8:e62765, 2013

31. Sitaram RT, Degerman S, Ljungberg B, Andersson E, Oji Y, Sugiyama H, et al: Wilms' tumour 1 can suppress hTERT gene expression and telomerase activity in clear cell renal cell carcinoma via multiple pathways. Br J Cancer 103:1255-1262, 2010

32. Steinert M, Wobus M, Boltze C, Schütz A, Wahlbuhl M, Hamann J, et al: Expression and regulation of CD97 in colorectal carcinoma cell lines and tumor tissues. Am J Pathol 161: 1657-1667, 2002

33. Stupp R, Mason WP, van den Bent MJ, Weller M, Fisher B, Taphoorn MJ, et al: Radiotherapy plus concomitant and adjuvant temozolomide for glioblastoma. N Engl J Med 352:987-996, 2005

34. Tatsumi N, Oji Y, Tsuji N, Tsuda A, Higashio M, Aoyagi S, et al: Wilms' tumor gene WT1-shRNA as a potent apoptosis-inducing agent for solid tumors. Int J Oncol 32:701-711, 2008

Manuscript submitted August 15, 2014.

Accepted September 23, 2014.

Please include this information when citing this paper: DOI: 10.3171/2014.9.FOCUS14506.

Address correspondence to: William C. Broaddus, M.D., Ph.D., Department of Neurosurgery, Box 980631, Virginia Commonwealth University School of Medicine, Richmond, VA 23219-0631. email: wbroaddus@mcvh-vcu.edu. 